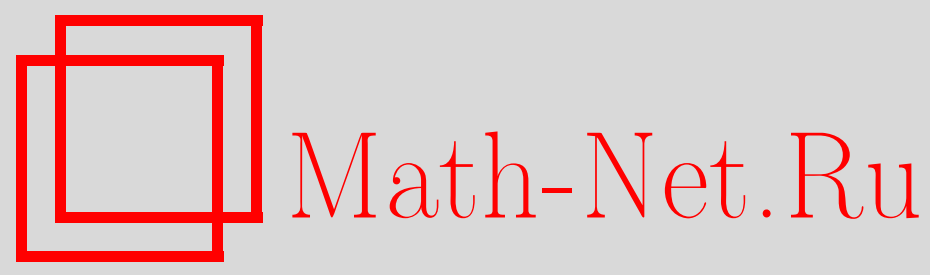

Ю. М. Нечепуренко, Интегральные критерии качества дихотомии спектра матрицы замкнутым контуром, Матем. заметки, 2005, том 78, выпуск 5, 718-726

DOI: https://doi.org/10.4213/mzm2635

Использование Общероссийского математического портала Math-Net.Ru подразумевает, что вы прочитали и согласны с пользовательским соглашением http://www . mathnet.ru/rus/agreement

Параметры загрузки:

IP : 52.87 .193 .239

26 апреля 2023 г., 15:54:44

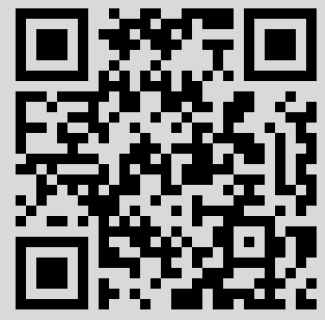




\title{
ИНТЕГРАЛЬНЫЕ КРИТЕРИИ КАЧЕСТВА ДИХОТОМИИ СПЕКТРА МАТРИЦЫ ЗАМКНУТЫМ КОНТУРОМ
}

Ю. М. Нечепуренко

\begin{abstract}
Известный метод оценки чувствительности инвариантных проекторов, основанньй на интегральных критериях качества дихотомии и ориентированный на возмущения общего вида, распространен на регулярно структурированные возмущения. Это позволяет, в частности, для конечномерных аппроксимаций дифференциальных операторов значительно точнее оценивать чувствительность инвариантных проекторов, отвечающих минимальным по модулю собственным значениям, к возмущению коэффициентов этих операторов. Дано новое определение интегрального критерия качества дихотомии, как некоторой $L_{p}$-нормы резольенты, что позволило упростить доказательства и сформулировать конечные результаты в более общем и простом виде.

Библиограффия: 7 названий.
\end{abstract}

1. Спектральньй анализ конечномерных линейных систем таких, как системы обыкновенных дифференциальных или разностных уравнений, приводит к матрицам вида

$$
P_{\gamma}(f, A)=\frac{1}{2 \pi i} \oint_{\gamma} f(z)(z I-A)^{-1} d z,
$$

где $A$ - квадратная матрица, $\gamma$ - некоторьй простой достаточно гладкий положительно ориентированньй замкнутьй контур, не проходяший через собственные значения матрицы $A, f$ - функция комплексного переменного, непрерьвная на контуре $\gamma$. При этом возникает задача оценки устойчивости таких матриц к возмущениям элементов матрицы $A$.

С. К. Годуновым (см., например, [1], [2]) были получены соответствующие оценки, основанные на

$$
m_{\gamma}(A)=\max _{z \in \gamma}\left\|(z I-A)^{-1}\right\|_{2}
$$

- максимуме нормы резольвенты на контуре $\gamma$, и оценки, основанные на интегральном критерии $\left\|H_{\gamma}(A)\right\|_{2}$ качества дихотомии спектра матрищы $A$ контуром $\gamma$, где

$$
H_{\gamma}(A)=\int_{\gamma}(z I-A)^{-*}(z I-A)^{-1}|d z| .
$$

Работа выполнена при финансовой поддержке Российского фонда фундаментальных исследований, грант № 04-01-00045, и программы РАН "Современные проблемы теоретической математики" (проект “Оптимизация вычислительных алгоритмов решения задач математической физики”). 
Эти оценки были получены для инвариантных проекторов, т.е. для случая $f(z) \equiv 1$, но легко переносятся на общий случай. Более серьезным препятствием, ограничивающим использование этих оценок, является их ориентация на возмущения общего вида, т.е. на случай, когда возмущенная матрица имеет вид $A+\Delta$, где $\Delta \in \mathbb{C}^{n \times n},\|\Delta\|_{2} \leqslant \varepsilon$, а $\varepsilon-$ заданная положительная величина. Для возмущений, имеющих специальный вид, эти оценки часто либо оказьваются слишком завышенными, либо вообще перестают работать, так как нарушается условие малости нормы возмущения, при котором они вьведены. Это имеет место, например, когда $A$ является конечномерной апшроксимацией некоторого дифференциального оператора, а возмущение $\Delta$ является возмущением его коэффициентов. В этом случае типична ситуация, когда величина $\varepsilon$ стремится к бесконечности при параметре дискретизации стремящемся к нулю и дажепри малом возмущении коэффициентов оценки из [1], [2] перестают работать. И, в тоже время, для контуров, охватывающих минимальные по модулю собственные значения, матрица $P_{\gamma}(f, A)$ мало отличается от $P_{\gamma}(f, A+\Delta)$ (именно эти собственные значения и отвечающие им матрицы $P_{\gamma}(f, A)$ представляют, как правило, наибольший практический интерес). Это кажущееся противоречие объясняется тем, что в данном случае мы имеем дело с возмущениями специального вида, оказьвающими наибольшее влияние на максимальные по модулю собственные значения и связанные с ними спектральные характеристики.

В работе [3] предлагается (без обоснования) обобщение оценок из [1], [2] на случай регулярно структурированных возмущений, т.е. возмущений $\Delta \in \mathbb{C}^{n \times n}$ таких, что $\left\|B^{-1} \Delta C^{-1}\right\|_{2} \leqslant \varepsilon$, где $B$ и $C \in \mathbb{C}^{n \times n}$ - фиксированные невырожденные матрицы, а $\varepsilon-$ заданная положительная величина. Обоснованию оценок из [3] посвящены п. 2 и п. 3 настоящей работы. В этих пунктах понятие интегрального критерия качества дихотомии определяется как некоторая $L_{p}$-норма резольвенты. Это позволяет упростить и упорядочить как доказательства, так и конечные результаты, и дает возможность использовать не только критерии качества дихотомии, аналогичные $m_{\gamma}(A)$ и $\left\|H_{\gamma}(A)\right\|_{2}$ и отвечающие соответственно $p=\infty$ и $p=2$, но и критерии, отвечающие любому $p \geqslant 1$.

В п. 4 на примере одномерного разностного оператора конвекции-диффузии демонстрируется, что новые оценки, со специально подобранными матрицами $B$ и $C$, значительно точнее старых в случае, когда $A$ - конечномерная аппроксимация дифференциального оператора, $\gamma$ - контур, охватывающий ее минимальные по модулю собственные значения, а возмущение вызвано возмушением коэффициентов этого оператора.

В п. 5 , как следствие одной из оценок, полученных в п. 2 , получены двусторонние оценки нормы сужения решения задачи Коши вида $u(0)=u^{0}, d u / d t=A u$ на инвариантное подпространство матрицы $A$, отвечающее ее собственным значениям, лежащим внутри контура $\gamma$.

2. Обозначим через $C(\gamma)^{k}$ линейное комплексное пространство непрерьвных функций $\gamma \rightarrow \mathbb{C}^{k}$ с нормами

$$
\|g\|_{\gamma, p}=\left(\int_{\gamma}\|g(z)\|_{2}^{p}|d z|\right)^{1 / p}, \quad 1 \leqslant p<\infty, \quad\|g\|_{\gamma, \infty}=\max _{z \in \gamma}\|g(z)\|_{2} .
$$

Через $C(\gamma)^{n \times n}$ обозначим линейное комплексное пространство квадратных матриц порядка $n$ с элементами из $C(\gamma)$, которое мы будем интерпретировать, как пространство 
линейных отображений $G: \mathbb{C}^{n} \rightarrow C(\gamma)^{n}$ с нормами

$$
\|G\|_{\gamma, p}=\max _{\|v\|_{2}=1}\|G v\|_{\gamma, p}, \quad v \in \mathbb{C}^{n}, \quad 1 \leqslant p \leqslant \infty
$$

Наряду с аксиомами норм линейного пространства и пространства линейных отображений, нормы $\|\cdot\|_{\gamma, p}$ в силу неравенства Гёльдера обладают следующим свойством. Для любой скалярной функции $f \in C(\gamma)$ и матрицы $G \in C(\gamma)^{n \times n}$ при всех $p \geqslant 1$ и $q=p /(p-1)$ имеем

$$
\|f G\|_{\gamma, 1}=\|f\|_{\gamma, q}\|G\|_{\gamma, p} .
$$

Кроме того, если $F$ - либо скалярная функция из $C(\gamma)$, либо матрица из $C(\gamma)^{n \times n}$, то

$$
\|F G\|_{\gamma, p}=\|F\|_{\gamma, \infty}\|G\|_{\gamma, p}
$$

a, если $F \in \mathbb{C}^{n \times n}$, то

$$
\|G F\|_{\gamma, p} \leqslant\|F\|_{2}\|G\|_{\gamma, p}
$$

Введем в рассмотрение величины

$$
\begin{aligned}
\kappa_{\gamma, p}(A ; B, C) & =\nu_{B, C}\left\|C(z I-A)^{-1} B\right\|_{\gamma, p} \\
& =\nu_{B, C} \max _{\|v\|_{2}=1}\left(\int_{\gamma}\left\|C(z I-A)^{-1} B v\right\|_{2}^{p}|d z|\right)^{1 / p}, \quad v \in \mathbb{C}^{n},
\end{aligned}
$$

где $\nu_{B, C}=\left\|B^{-1}\right\|_{2}\left\|C^{-1}\right\|_{2} /(2 \pi)$, которые будем назьвать интегральными критериями качества дихотомии спектра матрищы $A$ контуром $\gamma$. Здесь $p \geqslant 1$, а $B$ и $C-$ невырожденные матрицы. В частности, в соответствии с этим определением

$$
\kappa_{\gamma, \infty}(A ; B, C)=\nu_{B, C} \max _{z \in \gamma}\left\|C(z I-A)^{-1} B\right\|_{2}
$$

a

$$
\kappa_{\gamma, 2}(A ; B, C)=\nu_{B, C}\left\|\int_{\gamma} B^{*}(z I-A)^{-*} C^{*} C(z I-A)^{-1} B|d z|\right\|_{2}^{1 / 2} .
$$

ТЕорема 1. Имеем $\left\|P_{\gamma}(f, A)\right\|_{2} \leqslant\|f\|_{\gamma, q} \kappa_{\gamma, p}(A ; B, C)$.

ДоКАЗАТЕЛЬСТво. В силу (1)

$$
\begin{aligned}
\left\|P_{\gamma}(f, A)\right\|_{2} & \leqslant \frac{1}{2 \pi} \max _{\|v\|_{2}=1} \int_{\gamma}\left\|f(z)(z I-A)^{-1} v\right\|_{2}|d z| \\
& =\frac{1}{2 \pi}\left\|f(z)(z I-A)^{-1}\right\|_{\gamma, 1} \leqslant\|f\|_{\gamma, q} \kappa_{\gamma, p}(A ; I, I) .
\end{aligned}
$$

Осталось учесть, что в силу неравенств $(2)$ и $(3) \kappa_{\gamma, p}(A ; I, I) \leqslant \kappa_{\gamma, p}(A ; B, C)$. 
Теорема 2. Для любой матрицы $\Delta \in \mathbb{C}^{n \times n}$ maкой, что

$$
\omega=\left\|B^{-1} \Delta C^{-1}\right\|_{2} \kappa_{\gamma, \infty} \frac{(A ; B, C)}{\nu_{B, C}}<1,
$$

и всех $p \geqslant 1$ и $q=p /(p-1)$ справедливь следующие неравенства:

$$
\begin{aligned}
\left\|P_{\gamma}(f, A)-P_{\gamma}(f, A+\Delta)\right\|_{2} & \leqslant\|f\|_{\gamma, q} \kappa_{\gamma, p}(A ; B, C) \frac{\omega}{1-\omega}, \\
\left|\kappa_{\gamma, p}(A ; B, C)-\kappa_{\gamma, p}(A+\Delta ; B, C)\right| & \leqslant \kappa_{\gamma, p}(A ; B, C) \frac{\omega}{1-\omega} .
\end{aligned}
$$

ДокАЗАТЕЛЬСтво. В силу неравенств (1)-(3)

$$
\begin{aligned}
\left\|P_{\gamma}(f, A)-P_{\gamma}(f, A+\Delta)\right\|_{2} & \leqslant \frac{1}{2 \pi}\left\|f(z)\left[(z I-A)^{-1}-(z I-A-\Delta)^{-1}\right]\right\|_{1} \\
& \leqslant \frac{1}{2 \pi}\|f\|_{\gamma, q}\left\|(z I-A)^{-1}-(z I-A-\Delta)^{-1}\right\|_{\gamma, p} \\
& \leqslant\|f\|_{\gamma, q} \nu_{B, C}\left\|C(z I-A)^{-1} B-C(z I-A-\Delta)^{-1} B\right\|_{\gamma, p} .
\end{aligned}
$$

Нетрудно видеть, что

$$
(z I-A)\left[(z I-A)^{-1}-(z I-A-\Delta)^{-1}\right](z I-A-\Delta)=-\Delta,
$$

откуда

$$
\begin{aligned}
(z I-A)^{-1}-(z I-A-\Delta)^{-1} & =-(z I-A)^{-1} \Delta(z I-A-\Delta)^{-1} \\
& =-(z I-A)^{-1} \Delta\left(I-(z I-A)^{-1} \Delta\right)^{-1}(z I-A)^{-1}
\end{aligned}
$$

и, значит,

$$
\begin{aligned}
& C(z I-A)^{-1} B-C(z I-A-\Delta)^{-1} B \\
& \quad=-C(z I-A)^{-1} B S\left[I-C(z I-A)^{-1} B S\right]^{-1} C(z I-A)^{-1} B,
\end{aligned}
$$

где $S=B^{-1} \Delta C^{-1}$. Следовательно, если вьполнено условие (4), то в силу (2) имеем

$$
\begin{aligned}
& \nu_{B, C}\left\|C(z I-A)^{-1} B-C(z I-A-\Delta)^{-1} B\right\|_{\gamma, p} \\
& \quad \leqslant \nu_{B, C}\left\|C(z I-A)^{-1} B\right\|_{\gamma, p}\left\|C(z I-A)^{-1} B S\left[I-C(z I-A)^{-1} B S\right]^{-1}\right\|_{\gamma, \infty} \\
& \quad \leqslant \kappa_{\gamma, p}(A ; B, C) \frac{\omega}{1-\omega} .
\end{aligned}
$$

Подставляя (7) в (6), приходим к (5a).

Для доказательства (5b) достаточно заметить, что

$$
\begin{aligned}
& \left|\kappa_{\gamma, p}(A ; B, C)-\kappa_{\gamma, p}(A+\Delta ; B, C)\right| \\
& \quad=\nu_{B, C}\left|\left\|C(z I-A)^{-1} B\right\|_{\gamma, p}-\left\|C(z I-A-\Delta)^{-1} B\right\|_{\gamma, p}\right| \\
& \quad \leqslant \nu_{B, C}\left\|C(z I-A)^{-1} B-C(z I-A-\Delta)^{-1} B\right\|_{\gamma, p},
\end{aligned}
$$

и воспользоваться неравенством (7). 
3. Напомним, что структурированным псевдоспектром матрицы $A$ назьвают множество вида

$$
\Lambda_{\varepsilon}(A ; B, C)=\bigcup_{\|S\|_{2} \leqslant \varepsilon} \lambda(A+B S C), \quad \Delta \in \mathbb{C}^{n \times n},
$$

где $B$ и $C \in \mathbb{C}^{n \times n}$ - фиксированные матрицы, а $\lambda(\cdot)$ означает спектр матрицы [4], [5]. В частности, множество $\Lambda_{\varepsilon}(A)=\Lambda_{\varepsilon}(A ; I, I)$, где $I$ - единичная матрица порядка $n,-$ это обычный псевдоспектр матрищы $A[6]$. Структурированный псевдоспектр с невырожденньци матрицами $B$ и $C$ назьвают регулярно структурированным [7]. Для него справедливы следующие равенства:

$$
\Lambda_{\varepsilon}(A ; B, C)=\bigcup_{\left\|B^{-1} \Delta C^{-1}\right\|_{2} \leqslant \varepsilon} \lambda(A+\Delta), \quad \Delta \in \mathbb{C}^{n \times n},
$$

и

$$
\Lambda_{\varepsilon}(A ; B, C)=\left\{z:\left\|C(z I-A)^{-1} B\right\|_{2} \geqslant \frac{1}{\varepsilon}\right\}
$$

Условие (4) означает, что контур $\gamma$ не имеет общих точек с $\Lambda_{\varepsilon}(A ; B, C)$, где $\varepsilon=$ $\left\|B^{-1} \Delta C^{-1}\right\|_{2}$, т.е. осуществляет дихотомию этого регулярно структурированного псевдоспектра. В случае $p \neq \infty$ бьвает полезно переписать условие (4) так, чтобы единственной характеристикой матрицы $A$, фигурирующей в теореме 2 , был интегральньй критерий качества дихотомии $\kappa_{\gamma, p}(A ; B, C)$. Это можно сделать, оценив $\kappa_{\gamma, \infty}(A ; B, C)$ сверху через $\kappa_{\gamma, p}(A ; B, C)$ на основе следующих неравенств.

Теорема 3. Имеют место неравенства

$$
\begin{gathered}
\ln \left(1+\eta_{\infty}|\gamma|\right) \leqslant \eta_{1} \leqslant \eta_{\infty}|\gamma| \\
{\left[\frac{\eta_{\infty}^{p-1}}{p-1}\left(1-\frac{1}{\left(1+\eta_{\infty}|\gamma|\right)^{p-1}}\right)\right]^{1 / p} \leqslant \eta_{p} \leqslant \eta_{\infty}|\gamma|^{1 / p}, \quad p>1}
\end{gathered}
$$

где $\eta_{p}=\pi \kappa_{\gamma, p}(A ; B, C), a|\gamma|-$ длина контура $\gamma$.

Например, из левого неравенства (8) при $p=2$ можно получить следующую оценку:

$$
2 \eta_{\infty} \leqslant \eta_{2}^{2}+\left(\eta_{2}^{4}+\frac{4 \eta_{2}^{2}}{|\gamma|}\right)^{1 / 2}
$$

Это позволяет при любом фиксированном $p$ рассматривать интегральньй критерий качества дихотомии $\kappa_{\gamma, p}(A ; B, C)$, как меру устойчивости к соответствующим регулярно структурированньм возмущениям инвариантного подпространства, отвечающего собственньм значениям матрицы $A$, лежащим внутри контура $\gamma$. При этом, для выбора достаточно устойчивого инвариантного подпространства, полезно рассмотреть какое-нибудь однопараметрическое семейство контуров $\left\{\gamma_{r}: r_{1} \leqslant r \leqslant r_{2}\right\}$, например, систему вложенных окружностей, и построить график зависимости $\kappa_{\gamma_{r}, p}(A ; B, C)$ от $r$. Такую картинку назьвают одномерным спектральным портретом [1], [2]. 
ДОКАЗАТЕЛЬСТВО ТЕОРЕМЫ 3. Отметим прежде всего, что ограниченность $\eta_{p}$ при $p \geqslant 1$ влечет за собой ограниченность

$$
\eta_{\infty}=\pi \nu_{B, C} \max _{z \in \gamma}\left\|C(z I-A)^{-1} B\right\|_{2}
$$

Действительно, пусть резольвента неограничена в некоторй точке $z_{*} \in \gamma$. Тогда $z_{*}-$ собственное значение матрицы $A$. Обозначим через $v_{*}$ нормированный вектор такой, что $B v_{*}-$ собственньй вектор матрицы $A$, отвечающий $z_{*}$. Для $z \notin \lambda(A)$ имеем

$$
B^{-1}(z I-A) C^{-1} C B v_{*}=\left(z-z_{*}\right) v_{*} \Longrightarrow \frac{C B v_{*}}{z-z_{*}}=C(z I-A)^{-1} B v_{*}
$$

и, следовательно,

$$
\left\|C(z I-A)^{-1} B v_{*}\right\|_{2}=\frac{\left\|C B v_{*}\right\|_{2}}{\left|z-z_{*}\right|} .
$$

Последнее означает, что для достаточно гладкого контура $\gamma$, проходящего через точку $z_{*}$, выражение

$$
\max _{\|v\|_{2}=1}\left(\int_{\gamma}\left\|C(z I-A)^{-1} B v\right\|_{2}^{p}|d z|\right)^{1 / p} \geqslant\left(\int_{\gamma}\left\|C(z I-A)^{-1} B v_{*}\right\|_{2}^{p}|d z|\right)^{1 / p}
$$

не может быть ограничено при $p \geqslant 1$ и, следовательно, $\eta_{p}=\infty$.

Пусть $\eta_{p}<\infty$. Тогда $\eta_{\infty}<\infty$ и найдутся точка $z_{0} \in \gamma$ и нормированньй вектор $v_{0} \in \mathbb{C}^{n}$ такие, что

$$
\left\|B^{-1}\right\|_{2}\left\|C^{-1}\right\|_{2}\left\|C\left(z_{0} I-A\right)^{-1} B v_{0}\right\|_{2}=2 \eta_{\infty}
$$

Из тождества

$$
C(z I-A)^{-1} B-\left(z_{0}-z\right) C\left(z_{0} I-A\right)^{-1} B B^{-1} C^{-1} C(z I-A)^{-1} B=C\left(z_{0} I-A\right)^{-1} B
$$

следует, что

$$
\begin{aligned}
& \left\|C(z I-A)^{-1} B v_{0}\right\|_{2}+\left|z_{0}-z\right|\left\|B^{-1}\right\|_{2}\left\|C^{-1}\right\|_{2}\left\|C\left(z_{0} I-A\right)^{-1} B\right\|_{2}\left\|C(z I-A)^{-1} B v_{0}\right\|_{2} \\
& \quad \geqslant\left\|C\left(z_{0} I-A\right)^{-1} B v_{0}\right\|_{2},
\end{aligned}
$$

откуда

$$
\begin{aligned}
& \left\|B^{-1}\right\|_{2}\left\|C^{-1}\right\|_{2}\left\|C(z I-A)^{-1} B v_{0}\right\|_{2} \\
& \quad \geqslant \frac{\left\|B^{-1}\right\|_{2}\left\|C^{-1}\right\|_{2}\left\|C\left(z_{0} I-A\right)^{-1} B v_{0}\right\|_{2}}{1+\left\|B^{-1}\right\|_{2}\left\|C^{-1}\right\|_{2}\left\|C\left(z_{0} I-A\right)^{-1} B\right\|_{2}\left|z_{0}-z\right|}=\frac{2 \eta_{\infty}}{1+2 \eta_{\infty}\left|z_{0}-z\right|} .
\end{aligned}
$$

Пусть $s(z)$ при $z \in \gamma$ означает длину дуги контура $\gamma$, соединяющую точку $z$ с $z_{0}$. Поскольку $\left|z-z_{0}\right| \leqslant s(z)$,

$$
\left\|B^{-1}\right\|_{2}\left\|C^{-1}\right\|_{2}\left\|C(z I-A)^{-1} B v_{0}\right\|_{2} \geqslant \frac{2 \eta_{\infty}}{1+2 \eta_{\infty} s(z)}
$$


Обозначим через $\gamma_{1}$ и $\gamma_{2}$ дуги контура $\gamma$, соединяющие точку $z_{0}$ с самой отдаленной от нее точкой этого контура. Тогда $\gamma=\gamma_{1} \cup \gamma_{2},\left|\gamma_{1}\right|=\left|\gamma_{2}\right|=|\gamma| / 2$ и $|d z|=d s(z)$. Поэтому в силу (10)

$$
\begin{aligned}
\left(2 \eta_{p}\right)^{p} & =\left\|B^{-1}\right\|_{2}^{p}\left\|C^{-1}\right\|_{2}^{p} \max _{\|v\|_{2}=1} \int_{\gamma_{1} \cup \gamma_{2}}\left\|C(z I-A)^{-1} B v\right\|_{2}^{p} d s(z) \\
& \geqslant\left\|B^{-1}\right\|_{2}^{p}\left\|C^{-1}\right\|_{2}^{p} \int_{\gamma_{1} \cup \gamma_{2}}\left\|C(z I-A)^{-1} B v_{0}\right\|_{2}^{p} d s(z) \\
& \geqslant 2\left(2 \eta_{\infty}\right)^{p} \int_{0}^{|\gamma| / 2} \frac{d s}{\left(1+2 \eta_{\infty} s\right)^{p}} .
\end{aligned}
$$

Отсюда, взяв интеграл, получаем левые неравенства (8). Правые неравенства непосредственно следуют из неравенства (2) при $G=I$.

Отметим, что теоремы 1-3 и неравенство (9) обобщают аналогичные оценки из [1], [2], полученные для случаев $B=C=I, f(z) \equiv 1$ и $p=2$ либо $\infty$.

4. Покажем на примере оператора $L=d / d x+d^{2} / d x^{2}$, действующего на достаточно гладкие функции вещественного аргумента $x$, периодические с периодом 1 , что оценки, ориентированные на регулярно структурированные возмущения со специально подобранными матрицами $B$ и $C$, значительно точнее оценок, ориентированных на возмущения общего вида, когда $A$ - конечномерная аппроксимация дифференциального оператора, $\gamma$ - контур, охватьвающий ее минимальные по модулю собственные значения, а возмущение вызвано возмущением коэффициентов этого оператора.

Стандартная аптроксимация второго порядка точности центральными разностями в интервале $0<x<1$ на сетке с узлами $x_{j}=(2 j-1) /(2 n), j=1, \ldots, n$, приводит к разностному оператору, который может быть записан в матричном виде следующим образом: $A=F-D$, где $F$ и $D$ - квадратные матрицы порядка $n$ такие, что их $j$-е строки, умноженные на $n$-компонентный вектор $u$, - это соответственно

$$
(F u)_{j}=\frac{n\left(u_{j+1}-u_{j-1}\right)}{2}, \quad(D u)_{j}=n^{2}\left(-u_{j+1}+2 u_{j}-u_{j-1}\right)
$$

где $u_{0}=u_{n}$, a $u_{n+1}=u_{1}$. Число узлов сетки $n$ мы далее для определенности будем предполагать кратным 4.

Отметим, что матрицы $F$ и $D$ приводятся к диагональному виду унитарньг преобразованием подобия с матрицей

$$
\frac{1}{\sqrt{n}}\left\{\exp \left(i \frac{2 \pi k j}{n}\right)\right\}_{j, k=0}^{n-1}
$$

где $i$ означает мнимую единицу, а спектр матрицы $A$ - это множество всех

$$
\lambda_{l}=-4 n^{2} \sin ^{2} \frac{\pi l}{n}+i n \sin \frac{2 \pi l}{n}
$$

c $l \in L=\{0, \pm 1, \ldots, \pm(n / 2-1), n / 2\}$. 
Пусть $\gamma=\gamma_{r}$, где $\gamma_{r}$ - окружность радиуса $r$ с центром в начале координат, а $r$ удовлетворяет неравенству $\left|\lambda_{l_{0}}\right|<r<\left|\lambda_{l_{0}+1}\right|$ при некотором $l_{0}: 0 \leqslant l_{0}<n / 2$. И пусть нас интересуют возмущения $\Delta \in \mathscr{M}$, где

$$
\mathscr{M}=\left\{S F+F S: S=\operatorname{diag}\left(\delta_{1}, \ldots, \delta_{n}\right),\left|\delta_{j}\right| \leqslant \frac{\delta}{2}, 1 \leqslant j \leqslant n\right\},
$$

а $\delta$ - заданное положительное число.

Поскольку

$$
\max _{\Delta \in \mathscr{M}}\|\Delta\|_{2}=\|F\|_{2} \delta=n \delta,
$$

условие (4) при $B=C=I$ имеет вид

$$
\omega=\|\Delta\|_{2} \max _{z \in \gamma_{r}}\left\|(z I-A)^{-1}\right\|_{2}=n \rho \delta<1
$$

где

$$
\rho=\max \left\{\frac{1}{r-\left|\lambda_{l_{0}}\right|}, \frac{1}{\left|\lambda_{l_{0}+1}\right|-r}\right\} .
$$

Положим $B=C=(\alpha I+D)^{1 / 2}$, где $\alpha$-произвольное фиксированное положительное число. Тогда

$$
\max _{\Delta \in \mathscr{M}}\left\|B^{-1} \Delta C^{-1}\right\|_{2} \leqslant \frac{\delta}{\sqrt{\alpha}}
$$

a

$$
\max _{z \in \gamma_{r}}\left\|C(z I-A)^{-1} B\right\|_{2}=\max \left\{\frac{\alpha+\left|\operatorname{Re} \lambda_{l_{0}}\right|}{r-\left|\lambda_{l_{0}}\right|}, \frac{\alpha+\left|\operatorname{Re} \lambda_{l_{0}+1}\right|}{\left|\lambda_{l_{0}+1}\right|-r}\right\} \leqslant\left(\alpha+\left|\operatorname{Re} \lambda_{l_{0}+1}\right|\right) \rho .
$$

Поэтому условие (4) при $\gamma=\gamma_{r}$ и указанных вьше матрицах $B$ и $C$ вьполнено, если выполнено неравенство

$$
q \rho \delta<1
$$

где $q=\left(\alpha+4 n^{2} \sin ^{2}\left(\pi\left(l_{0}+1\right) / n\right)\right) / \sqrt{\alpha}$. Для любого $l_{0}: 0 \leqslant l_{0} \ll n$, выбрав подходящее значение $\alpha$, мы получим значительно более мягкое условие, чем (11). Например, положив $\alpha=4 \pi^{2}\left(l_{0}+1\right)^{2}$, мы получим условие $(12)$ с $q \leqslant 4 \pi\left(l_{0}+1\right)$.

5. Пусть $f(z)=e^{t z}$. Тогда $P_{\gamma}(f, A)=e^{t A} P_{\gamma}(A)$, где $P_{\gamma}(A)$ - инвариантный проектор матрищы $A$, отвечающий ее собственным значениям, лежашим внутри контура $\gamma$. Теорема 1 позволяет получать двусторонние оценки нормы вектора $e^{t A} P_{\gamma}(A) u^{0}$ через норму вектора $P_{\gamma}(A) u^{0}$ для произвольного вектора $u^{0} \in \mathbb{C}^{n}$.

Лемма 1. Пусть при всех вещественных $t$ справедливо неравенство

$$
\left\|e^{t A} P_{\gamma}(A)\right\|_{2} \leqslant \varphi(t)
$$

где $\varphi(t)$ - некоторая полохительная функиия от $t$. Тогда для всех $u^{0} \in \mathbb{C}^{n}$ и всех вещественных $t$ справедливы неравенства

$$
\frac{\left\|P_{\gamma}(A) u^{0}\right\|_{2}}{\varphi(-t)} \leqslant\left\|e^{t A} P_{\gamma}(A) u^{0}\right\|_{2} \leqslant\left\|P_{\gamma}(A) u^{0}\right\|_{2} \varphi(t) .
$$


Действительно, правое неравенство (14) непосредственно следует из (12). Левое неравенство (14) получаем из правого, заменив в нем $u^{0}$ на $e^{-t A} u^{0}, t$ на $-t$ и разделив полученное неравенство на $\varphi(-t)$.

Поскольку в силу теоремы 1 неравенство (13) справедливо при всех вещественных $t$ с

$$
\varphi(t)=\left(\int_{\gamma} e^{q \operatorname{Re} z t}|d z|\right)^{1 / q} \kappa_{\gamma, p}(A ; B, C)
$$

в силу леммы 1 с этим же $\varphi(t)$ справедливы неравенства (13).

Отметим, что вектор $e^{t A} P_{\gamma}(A) u^{0}$ является сужением решения задачи Коши $u(0)=u^{0}$, $d u / d t=A u$ на инвариантное подпространство матрицы $A$, отвечающее ее собственным значениям, лежащим внутри контура $\gamma$.

\section{СПИСОК ЦИТИРОВАННОЙ ЛИТЕРАТУРЫ}

[1] Годунов С. К. Современные аспекты линейной алгебры. Новосибирск: Научная книга, 1997.

[2] Годунов С. К. Лекции по современным аспектам линейной алгебры. Новосибирск: Научная книга, 2002.

[3] Нечепуренко Ю. М. О дихотомии спектра матрицы замкнутым контуром // Докл. РАН. 2004. T. 397. № 4. C. 459-460.

[4] Hinrichsen D., Kelb B. Spectral value sets: a graphical tool for robustness analysis // Systems Control Lett. 1993. V. 21. P. 127-136.

[5] Gallestay E., Hinrichsen D., Pritchard A. J. Spectral value sets of closed linear operators // Proc. Roy. Soc. London Ser. A. 2000. V. 456. P. 1397-1418.

[6] Trefethen L. N. Pseudospectra of linear operators // SIAM Review. 1997. V. 39. P. 383-406.

[7] Nechepurenko Yu. M. The regularly structured pseudospectrum // Russ. J. Numer. Anal. Math. Modelling. 2004. V. 19. № 3. P. 265-268. 